\title{
Estratégia ótima para declarar perdas no mercado de seguros de automóveis
}

\author{
Bruno C. A. Ledo \\ Professor - Universidade de São Paulo ( FEA-USP/RP) \\ Endereço: Av. Bandeirantes, 3900 - Campus da USP - Ribeirão Preto/SP \\ CEP: 14040-900 -E-mail: bruno@fearp.usp.br

\section{Mariana F. Boreli} \\ Doutoranda - Escola de Economia de São Paulo - Fundação Getúlio Vargas (EESP-FGV) \\ Endereço: Rua Itapeva, 474 - Bela Vista - São Paulo \\ CEP: 01313-902 - E-mail: mariana.boreli@gmail.com
}

Recebido: 26/06/2015. Aceite 23/11/2016.

\section{Resumo}

Ao analisar uma extensa base de dados sobre o mercado brasileiro de seguros de automóveis, encontramos um fato estilizado: a frequência de sinistros declarados é maior nos primeiros meses de vigência contratual. Para explicá-lo, propomos uma versão modicada do modelo de Venezia e Levy (1980), na qual demonstramos que a pseudofranquia é menor no início do contrato. A pseudofranquia é um valor não observável, superior à franquia estipulada em contrato, até o qual o segurado tem incentivo a não declarar suas perdas. Dado que a pseudofranquia é menor no início do contrato, o modelo prevê que haverá uma frequência maior de perdas (de menor valor) sendo declaradas neste período. Para testar esta previsão, regredimos o logaritmo das indenizações contra dummies de tempo, e obtivemos que no primeiro trimestre de vigência o valor médio das perdas declaradas é aproximadamente 2,21\% menor em relação ao segundo trimestre. Esta informação pode ser utilizada pelas seguradoras para ajustar o valor das franquias nos primeiros meses dos contratos a fim de reduzir suas taxas de sinistralidade.

\section{Palavras-Chave}

Seguro de automóvel. Pseudofranquia. Declarar sinistro.

\begin{abstract}
Upon analyzing an extensive database on the Brazilian auto insurance market, we found a stylized fact: the frequency of claims is higher in the first months of the contract. In order to explain this fact, we propose a modified version of Venezia and Levy (1980) model, in which we show that pseudodeductible is lower at the beginning of the contract. The pseudodeductible is an unobservable threshold, higher than the deductible stipulated in the contract, below which the insuree has an incentive not to claim his losses. Given that the pseudodeductible is smaller at the beginning of the contract, the model predicts that there will be a higher frequency of losses (of lower value) being claimed in this period. In order to test this prediction, we regressed the logarithm of
\end{abstract}

\footnotetext{
- Agradecemos ao CNPq por ter possibilitado e financiado esta pesquisa.
} 
indemnities against dummies that indicate the time of the contract, and we found that in the first trimester of the contract the average value of claimed losses is approximately $2.21 \%$ lower than the second trimester. This information can be used by insurers to adjust deductibles in the first months of contracts in order to reduce their claim rates.

\section{Keywords}

Auto insurance. Pseudeductible. Claim.

\section{JEL Classification}

D1. C10.

\section{Introdução}

Ao explorar uma extensa base de dados sobre o mercado brasileiro de seguros de automóveis, identificamos o seguinte fato estilizado: a frequência de sinistros declarados é significativamente maior nos primeiros meses de vigência contratual. ${ }^{1} \mathrm{O}$ presente artigo tem como objetivo compreender o racional econômico por trás desta evidência empírica. A princípio, este fato estilizado poderia ser explicado de diferentes maneiras.

Pode-se argumentar que os indivíduos menos experientes tendem a melhorar suas habilidades ao volante com o passar do tempo, logo, apresentariam menos acidentes ao final do contrato. No entanto, mesmo nas subamostras de segurados mais experientes o fato estilizado ainda persistia de modo significativo.

Outro argumento plausível aponta que o crescimento natural da economia faria com que mais contratos de seguros fossem vendidos com o passar do tempo. Sendo assim, na amostra completa haveria um número maior de contratos em seus primeiros meses de vigência. Portanto, se há mais apólices, também deve haver mais sinistros. Para eliminar este potencial viés, analisamos subamostras de contratos vendidos em um mesmo mês, e verificamos que o fato estilizado ainda permanecia.

\footnotetext{
1 No mercado de seguros de automóveis, o termo "sinistro" é utilizado para designar qualquer
} evento em que haja perda material sobre o veículo assegurado. 
O terceiro argumento, que será explorado neste trabalho, aponta que os segurados simplesmente respondem aos incentivos implícitos nos contratos de seguro. Deste modo, mecanismos como franquia e o sistema de bônus seriam os verdadeiros responsáveis por observarmos mais perdas sendo declaradas no início da vigência.

A franquia é um instrumento utilizado pelas seguradoras para minimizar o risco moral. Em caso de sinistro, as seguradoras se comprometem a indenizar apenas a diferença entre o valor da perda e o da franquia. Sendo assim, prejuízos inferiores ao valor franqueado não seriam declarados pelos segurados. ${ }^{2}$ Também para reduzir o risco moral, as seguradoras utilizam um sistema intitulado bonus-malus, no qual penalizam os segurados que declaram acidentes, com acréscimos sobre valor do prêmio em uma eventual renovação contratual, e bonificam aqueles que não acionaram o seguro, com descontos sobre o preço da apólice. Dessa forma, ao sofrer um sinistro de perda parcial, o segurado enfrenta um trade-off entre declará-lo ou não à seguradora. Caso opte por declarar, ele terá mais riqueza no presente e menos no futuro. No presente, receberá a indenização da seguradora, porém, no futuro sofrerá um acréscimo sobre o prêmio (malus). Por outro lado, caso opte por não declarar, ele terá menos riqueza no presente e mais no futuro. No presente o segurado pagará sua perda integralmente, no entanto, receberá um desconto sobre o prêmio do seguro no futuro (bonus).

Dado este trade-off, é possível que o segurado escolha não comunicar à seguradora certas perdas, ainda que estas sejam superiores ao valor da franquia estipulada em contrato. Caso a diferença entre a franquia e o valor da perda seja suficientemente pequena em relação ao valor presente dos descontos (bonus) nas apólices futuras, o segurado não terá incentivo a declarar o sinistro. O termo pseudofranquia foi cunhado por Braun et al. (2006) para designar o valor (latente) máximo de perda até o qual o segurado escolherá não acionar o seguro.

É importante destacar que esse trade-off se torna ainda mais complexo ao considerar o timing de ocorrência do sinistro, pois quanto mais próximo do início do contrato, maior a incerteza em relação à ocorrência de novas perdas no decorrer da mesma apólice. Assim,

2 A legislação brasileira só permite cobrança de franquia em caso de colisão parcial. Esta não é cobrada em caso de colisão total, roubo ou furto. 
o problema do segurado se resume a uma escolha envolvendo risco, cuja solução dependerá de uma relação complexa entre o valor da franquia, os valores de bonus-malus, o timing do acidente, a aversão ao risco do segurado, sua taxa de desconto intertemporal e sua distribuição de perdas.

Neste artigo, apresentamos uma versão modificada do modelo desenvolvido por Venezia e Levy (1980) e derivamos a pseudofranquia a partir da condição de indiferença entre declarar ou não um sinistro. É importante destacar que nosso modelo se diferencia do original por permitir que o contrato dure por dois períodos. Como consequência, nosso modelo incorpora o timing de decisão do segurado e possibilita que haja mais de um sinistro em um mesmo contrato.

O modelo original proposto por Venezia e Levy (1980) considera que cada contrato dura apenas um período, então não faria sentido falarmos em perdas declaradas no "início" do contrato. Daí a importância de flexibilizarmos o modelo para permitir que um contrato dure dois períodos. Dada a existência do sistema de bônus, esta pequena mudança terá consequências não triviais.

Venezia e Levy (1980) preveem que a pseudofranquia será monotonicamente decrescente com o passar do tempo (de um contrato para outro). Nossa versão modificada do modelo também chega nesse resultado, porém, adicionalmente conseguimos demonstrar que a pseudofranquia deverá aumentar do primeiro para o segundo período de um mesmo contrato. Logo, se a pseudofranquia é menor no início do contrato, haverá uma frequência maior de perdas (de menor valor) sendo declaradas neste período, o que explica o fato estilizado observado nos dados brasileiros.

Para testar esta previsão, regredimos o logaritmo das indenizações contra dummies referentes ao período de vigência do contrato, um conjunto de outras variáveis observáveis e dummies referentes a cada mês do primeiro semestre de 2004. Os resultados obtidos corroboram a previsão do modelo. Por exemplo, obtivemos que no primeiro trimestre de vigência contratual o valor médio dos sinistros comunicados à seguradora é aproximadamente 2,21\% menor em relação ao segundo trimestre. Além disso, no primeiro quadrimestre as perdas declaradas são aproximadamente $2,45 \%$ menores do que no segundo quadrimestre. Tais informações podem ser utilizadas pelas segura- 
doras para ajustar o valor das franquias nos primeiros meses dos contratos com o objetivo de reduzir suas taxas de sinistralidade.

O restante deste artigo está dividido conforme segue: na Seção 2 apresentamos uma revisão da literatura; na Seção 3 descrevemos a base de dados e apontamos algumas estatísticas descritivas que constatam a existência do fato estilizado; na Seção 4 desenvolvemos o modelo para o problema de escolha dinâmica sob incerteza do segurado; na Seção 5 recriamos o exemplo numérico proposto por Venezia e Levy (1980); na Seção 6 testamos e corroboramos a principal previsão do modelo; na Seção 7, apresentamos as conclusões.

\section{Literatura relacionada}

A decisão de declarar ou não um sinistro é estudada desde os anos 50. Embora o termo pseudofranquia ainda não tivesse sido cunhado, os primeiros modelos já consideravam que os segurados só teriam incentivo a declarar perdas que superassem um determinado valor crítico, conforme Grenander (1957) e Lanzenauer (1969; 1972a,b). No entanto, estes autores assumem que os segurados são neutros ao risco, de modo que o valor crítico para declarar perdas é determinado pela minimização de custos de longo prazo. Na literatura mais recente, a hipótese de neutralidade ao risco é pouco utilizada para modelar decisões de consumidores diante do risco, sendo mais utilizada para modelar decisões de empresas que operam em ambientes onde a hipótese da Lei dos Grandes Números pareça mais plausível.

Tal como em nosso modelo, De Leve e Weeda (1968) consideram que a decisão do segurado depende do momento contratual, quando a perda ocorre, e do valor do prêmio pago. O modelo desses autores também permite que haja mais de um sinistro durante a vigência contratual, e considera o timing de ocorrência das perdas, mas não demonstra que os segurados teriam maior incentivo a declarar sinistros no início do contrato. Isto porque os autores assumem que ao comunicar um acidente à seguradora, em qualquer momento, o segurado será penalizado com a maior classe de risco possível ao renovar o contrato. Assim, depois de acionar o seguro pela primeira vez, todos as perdas subsequentes seriam declaradas (caso equivalente ao último período do nosso modelo). 
À medida que a literatura foi se desenvolvendo, o trade-off enfrentado pelo segurado diante de um sinistro foi se tornando cada vez mais detalhado. Lanzenauer (1974) o descreveu da seguinte forma: "na presença de um sistema de rating de risco, o segurado, ao incorrer em uma perda, decidirá entre declará-la e aceitar o aumento futuro no prêmio ou arcar sozinho com a perda e melhorar seu rating de risco". O autor desenvolve um modelo dinâmico e encontra uma regra ótima de decisão para esse problema tal que essa é função da classe de risco, do número de acidentes declarados e do momento em que a decisão é tomada (timing). No entanto, o autor considera que o segurado é neutro ao risco. Nesta mesma linha teórica, e também supondo neutralidade ao risco, encontram-se os modelos propostos por Lemaire (1996), Norberg (1976) e De Pril (1979). Embora nosso modelo se assemelhe com esta corrente teórica em muitos aspectos, assumimos que os segurados são avessos ao risco, especialmente porque em nosso modelo o horizonte de tempo é finito e os segurados compram um único contrato por período.

O modelo proposto por Hastings (1976) considera que o valor crítico da regra de decisão do segurado é constante durante o período contratual, e, portanto, prevê uma quantidade uniforme de sinistros declarados ao longo do ano. Inaugurando uma nova corrente teórica, Norman e Shearn (1980) flexibilizam a hipótese de que o valor crítico da perda é constante ao longo do contrato, e apresentam uma versão simplificada do modelo do Lanzenauer (1974), propondo uma regra de ouro simples: uma perda será declarada apenas se exceder a diferença entre o total de prêmios a pagar nos próximos quatro anos se um acidente é declarado e o valor total caso nenhum sinistro seja comunicado à seguradora, assumindo que nenhuma perda adicional ocorre.

Um outro aspecto importante da decisão do segurado foi incorporado por Venezia e Levy (1980) e Kolderman e Volgenant (1985) ao considerarem que um acidente declarado é suficiente para alterar a classe de risco do segurado. Porém, Kolderman e Volgenant (1985) supõem que as perdas que ocorrem no tempo restante do contrato não têm efeito nos custos esperados. 
Dellaert et al. (1990) dividem a vigência contratual em $\mathrm{N}$ períodos que são iguais e independentes; além disso, ocorre no máximo uma perda no período e o prêmio é pago no início de cada ano. Este é o único artigo que encontramos na literatura que permite comparar a pseudofranquia intra e intercontratos. Apesar de este artigo racionalizar nosso fato estilizado, três aspectos importantes diferenciam a nossa abordagem teórica desses autores: (i) consideramos segurados avessos ao risco; (ii) o segurado é penalizado quando declara mais de um sinistro; (iii) o comportamento da pseudofranquia intercontratos é diferente.

Ao contrário da literatura citada anteriormente, Venezia e Levy (1980) consideram que o segurado é avesso ao risco, e que a sua decisão não está pautada apenas na minimização do valor presente dos custos. Assim, propõem um modelo recursivo que determina a estratégia ótima do problema de declarar ou não um sinistro ao igualar o valor presente da utilidade esperada ao acionar o seguro ao valor desta quando o acidente não é comunicado à seguradora. Com isso, os autores encontram um valor crítico para as perdas tal que o segurado é indiferente entre declarar ou não o sinistro. Esse determina a regra ótima de decisão: perdas superiores a ele serão declaradas. No entanto, o modelo não permite que haja mais de um sinistro na mesma apólice, portanto, não é capaz de explicar porque observamos uma frequência maior de acidentes declarados no início da vigência contratual.

Outra característica relevante é adicionada ao modelo anterior por Venezia (1984) ao analisar simultaneamente a escolha da apólice (cobertura) e a decisão de declarar ou não um sinistro. ${ }^{3} \mathrm{O}$ autor infere que o critério que determina se no presente o segurado comunicará a ocorrência de um acidente à seguradora depende fundamentalmente do contrato que deseja obter no futuro, afinal, o desconto (ou acréscimo) do sistema bonus-malus incidirá sobre o prêmio da apólice que será escolhida no próximo período.

3 Cobertura refere-se à proteção contratada pelo segurado. 
Toda literatura citada anteriormente, assim como nosso modelo, assume informação completa por parte dos agentes, ou seja, os segurados sabem exatamente o número de acidentes, as perdas correspondentes e os valores futuros dos prêmios. De Pril e Goovaerts (1983) são os únicos que utilizam informação incompleta para calcular limites para a pseudofranquia.

A partir de uma base de dados que contém informaçõess sobre seguros de imóveis, Braun et al. (2006) mostram que o número de sinistros observados pode ser modelado como o resultado de três processos estocásticos relevantes: a taxa de ocorrência das perdas, a magnitude de cada uma dessas e a escolha do segurado de acionar ou não o seguro. Adicionalmente, o autor utiliza inferência Bayesiana para identificar os acidentes que não foram declaradas e cria o termo pseudofranquia.

Kerr (2012) transfere este conceito para o mercado de seguro de automóveis e estima um modelo logit para determinar a propensão a declarar uma perda relativa à decisão de não fazê-la em função da presença da pseudofranquia que decorre de fatores primários identificados por Braun et al. (2006), que são o tamanho da perda econômica e o histórico de declarações do segurado. A implementação de tal metodologia só foi possível porque o autor teve acesso a uma base de dados contendo informações sobre os sinistros não declarados. Apenas estes dois artigos têm como objetivo estimar a pseudofranquia.

O ponto fundamental que diferencia a nossa abordagem teórica da literatura aqui citada é a possibilidade de ocorrer mais de um acidente em um mesmo contrato, com segurados avessos ao risco. Lanzenauer (1974), De Leve e Weeda (1968), Dellaert et al. (1990) permitem várias perdas durante a vigência de uma apólice de seguro, no entanto, consideram agentes neutros ao risco. Por outro lado, Venezia e Levy (1980) consideram um segurado avesso ao risco, porém, não permitem múltiplos acidentes no mesmo período contratual. Assim, ao incorporar esta hipótese neste modelo, conseguimos destacar o papel do timing da ocorrência do sinistro, de modo que nos permite explorar o comportamento da pseudofranquia intra e intercontratos. 


\section{Base de dados}

A base de dados utilizada neste artigo foi elaborada pela Superintendência de Seguros Privados (SUSEP). Trata-se de um corte transversal contendo a população das apólices de seguros de automóveis que estiveram vigentes no Brasil, ao menos por um dia, durante o primeiro semestre de $2004 .{ }^{4}$ Vale enfatizar que os dados estão ao nível da apólice, sendo assim, para cada contrato, observamos as coberturas, os prêmios e sinistros declarados, além de informações sobre as características dos veículos e dos segurados.

Para fins de homogeneidade amostral, optamos por trabalhar com uma subamostra de contratos com cobertura compreensiva (total) para veículos de passeio. Excluímos também apólices de pessoa jurídica, coletivas ou que sofreram qualquer tipo de alteração durante o período de vigência. Finalmente, dado nosso interesse em estudar o trade-off ao declarar uma perda, decidimos retirar da amostra os contratos que não possuíam franquia. ${ }^{5}$ Ao final, nossa subamostra ficou com 6,6 milhões de observações. A Tabela 1 apresenta todas as variáveis contidas na base de dados.

4 Dado que as apólices têm validade anual, nossa amostra contém contratos que foram transacionados entre 01/01/2003 e 30/06/2004, por todas as seguradoras, em todo território nacional. Só não constam na amostra os contratos que, por algum motivo, foram cancelados antes de $01 / 01 / 2004$.

5 Estes representavam aproximadamente $1 \%$ do total. 


\section{Tabela 1 - Descrição das variáveis}

\begin{tabular}{|c|c|}
\hline Variável & Descrição \\
\hline Apólice & $\begin{array}{l}\text { Variável que corresponde ao número da apólice. Esta é a principal chave de } \\
\text { identificação de um contrato, pois cada uma possui um número. }\end{array}$ \\
\hline Prêmio do casco & $\begin{array}{l}\text { Variável que corresponde ao valor do prêmio, pago pelo segurado à segura- } \\
\text { dora, relativo à cobertura de casco (veículo). }\end{array}$ \\
\hline Código do modelo & Variável alfanumérica que designa o modelo do veículo. \\
\hline Ano do modelo & $\begin{array}{l}\text { Corresponde ao ano do veículo. No modelo econométrico foram considerados } \\
\text { os veículos fabricados entre } 1993 \text { e } 2005 \text {. }\end{array}$ \\
\hline Região geográfica & $\begin{array}{l}\text { Variável numérica entre } 1 \text { e } 40 \text {. Cada valor corresponde a uma região difer- } \\
\text { ente no Brasil. }\end{array}$ \\
\hline Tipo de cobertura & $\begin{array}{l}\text { Variável numérica que descreve o tipo de cobertura contratada de modo que } \\
\text { o código } 1 \text { representa a cobertura compreensiva; } 2 \text { para cobertura contra } \\
\text { incêndio e roubo; } 3 \text { apenas para incêndio; } 4 \text { para perda total, colisão e roubo; } \\
\text { e, } 9 \text { para outras. Consideramos na análise apenas a cobertura compreensiva. }\end{array}$ \\
\hline Tipo de franquia & $\begin{array}{l}\text { Variável numérica que corresponde ao tipo de franquia contratada dentre elas } \\
\text { a reduzida, a normal, a majorada e a sem franquia. Essas representam os } \\
\text { valores } 1,2,3 \text {, e } 9 \text { respectivamente. Neste artigo consideramos as franquias } \\
\text { do tipo } 1 \text { e } 2 \text {. }\end{array}$ \\
\hline Valor da franquia & $\begin{array}{l}\text { Valor estabelecido no contrato até o qual o segurado não receberá indeni- } \\
\text { zação em caso de sinistro. }\end{array}$ \\
\hline Data de nascimento & Corresponde a data de nascimento do principal condutor do veículo. \\
\hline Indenização & $\begin{array}{l}\text { Variável que corresponde ao valor total da indenização, paga pela seguradora } \\
\text { ao segurado, caso ocorra sinistro de casco. }\end{array}$ \\
\hline Sexo & Variável que corresponde ao sexo do principal condutor do veículo. \\
\hline Porcentagem de corretagem & $\begin{array}{l}\text { Variável que corresponde à taxa de corretagem (sobre o prêmio do seguro) } \\
\text { recebida pelo corretor em função da venda do contrato. }\end{array}$ \\
\hline Porcentagem de bônus & $\begin{array}{l}\text { Variável que corresponde ao percentual de desconto (bônus) sobre o prêmio } \\
\text { total do casco, concedido ao segurado pela não ocorrência de sinistro de } \\
\text { casco em contratos anteriores. }\end{array}$ \\
\hline Início da vigência & Variável que corresponde à data de início da vigência da apólice. \\
\hline Fim da vigência & Variável que corresponde à data de término da vigência da apólice. \\
\hline Importância do casco & $\begin{array}{l}\text { Variável que corresponde ao valor da importância segurada contratada para } \\
\text { cobertura de casco. }\end{array}$ \\
\hline Categoria tarifária & $\begin{array}{l}\text { Variável que corresponde ao código tarifário em que o veículo se enquadra. } \\
\text { Há } 8 \text { categorias e consideramos apenas a categoria passeio nacional. }\end{array}$ \\
\hline
\end{tabular}

Fonte: Elaboração própria com base nas informações fornecidas pela SUSEP.

A Tabela 2 mostra a frequência mensal de sinistros de perda parcial declarados no primeiro semestre de 2004. Nota-se que houve um aumento no número de acidentes comunicados à seguradora entre janeiro e maio de 2004, seguido de uma leve redução em junho. No total, os segurados acionaram o seguro 138.839 vezes. $^{6}$

${ }^{6}$ Consideramos apenas o primeiro acidente de perda parcial declarado para cada apólice. 
Tabela 2 - Frequência de sinistros declarados

\begin{tabular}{cc}
\hline Mês calendário & Sinistros declarados \\
\hline Janeiro & 21.242 \\
Fevereiro & 21.348 \\
Marco & 23.329 \\
Abril & 23.461 \\
Maio & 25.742 \\
Junho & 23.717 \\
\hline Total de sinistros & 138.839 \\
\hline
\end{tabular}

Fonte: Elaboração própria.

Todas as apólices de seguro da nossa amostra duram 366 dias. Sendo assim, dividimos o tempo de vigência de cada contrato em 12 partes iguais, contendo 30,5 dias cada, e chamamos estas partes de "mês de vigência". É importante salientar que as definimos como um conjunto de 30,5 dias contíguos, ou seja, nossa definição de mês de vigência não necessariamente coincide com o mês do calendário (janeiro, fevereiro, etc).

A quantidade de acidentes declarados em cada mês de vigência pode ser observada na segunda coluna da Tabela 3. Nota-se que dos 138.839 acidentes que foram comunicados às seguradoras no primeiro semestre de 2004, 14.269 ocorreram no primeiro mês de vigência, 13.911 no segundo mês de vigência, e assim por diante. Esta coluna também apresenta nosso fato estilizado: a frequência de sinistros declarados é maior no início do contrato, e decresce à medida que o contrato se aproxima do término. Nota-se que o número de perdas comunicadas à seguradora no primeiro mês de vigência é $48 \%$ maior do que as declaradas no último mês da apólice. 
Tabela 3 - Sinistros declarados de acordo com o mês de vigência, considerando várias subamostras

\begin{tabular}{|c|c|c|c|c|c|}
\hline \multicolumn{6}{|c|}{ Quantidade de sinistros declarados } \\
\hline \multirow{2}{*}{$\begin{array}{l}\text { Mês de } \\
\text { vigência }\end{array}$} & \multirow[b]{2}{*}{ No semestre } & \multirow[b]{2}{*}{ Em janeiro } & \multirow{2}{*}{$\begin{array}{l}\text { Em janeiro e idade } \\
\text { superior a } 25 \text { anos }\end{array}$} & \multicolumn{2}{|c|}{ Ocorreram em janeiro e idade superior a 25 anos } \\
\hline & & & & $\begin{array}{l}\text { Modelo do veículo } \\
\text { anterior a } 2003\end{array}$ & $\begin{array}{l}\text { Porcentagem de bônus } \\
\text { positiva }\end{array}$ \\
\hline $1^{0}$ & 14.269 & 2.034 & 1.900 & 1.445 & 1.379 \\
\hline $2^{0}$ & 13.911 & 2.140 & 2.002 & 1.479 & 1.499 \\
\hline $3^{0}$ & 12.537 & 1.781 & 1.673 & 1.228 & 1.288 \\
\hline $4^{0}$ & 12.539 & 1.867 & 1.732 & 1.336 & 1.345 \\
\hline $5^{0}$ & 11.468 & 1.726 & 1.603 & 1.293 & 1.251 \\
\hline $6^{0}$ & 11.436 & 1.771 & 1.675 & 1.391 & 1.276 \\
\hline $7^{0}$ & 11.049 & 1.773 & 1.678 & 1.403 & 1.299 \\
\hline $8^{0}$ & 10.951 & 1.720 & 1.619 & 1.362 & 1.259 \\
\hline$g^{0}$ & 10.377 & 1.614 & 1.517 & 1.280 & 1.161 \\
\hline $10^{\circ}$ & 10.601 & 1.680 & 1.568 & 1.323 & 1.175 \\
\hline $11^{\circ}$ & 10.033 & 1.577 & 1.482 & 1.277 & 1.148 \\
\hline $12^{0}$ & 9.668 & 1.559 & 1.469 & 1.273 & 1.127 \\
\hline Total & 138.839 & 21.242 & 19.918 & 16.090 & 15.207 \\
\hline
\end{tabular}

Fonte: Elaboração própria.

Uma questão relevante que se pode levantar é: como a inflação poderia influenciar os resultados apresentados na segunda coluna da Tabela 3? Ceteris paribus, à medida que o tempo passa, maior o custo de conserto de um veículo. Assim, dado um valor fixo da franquia, o efeito inflacionário tornaria a diferença entre o custo de conserto e o valor da franquia cada vez maior, incentivando os segurados a declararem tais perdas. De acordo com esta lógica, devemos observar mais sinistros sendo declarados no final da vigência contratual. Porém, mesmo na presença deste potencial viés, a frequência de perdas é maior no início dos contratos.

Com o intuito de minimizar este possível viés causado pela inflação, na terceira coluna da Tabela 3 fixamos o mês de ocorrência das perdas (em janeiro de 2004). Podemos observar que, dentre todos os 21.242 acidentes que foram declarados em janeiro, 2.034 ocorreram durante o primeiro mês de vigência, e, novamente, esse valor cai aproximadamente em $25 \%$ quando comparado com o $12^{\circ}$ mês de vigência. ${ }^{7}$ Além da pseudofranquia, escopo deste trabalho, outros dois fatores poderiam explicar a maior frequência de sinistros de-

7 Ao aplicarmos a mesma metodologia para analisar os meses de fevereiro, março, abril, maio e junho de 2004, constatamos o mesmo fato estilizado. 
clarados no início da vigência contratual: (i) o efeito aprendizado dos segurados; e, (ii) o crescimento no número de contratos vendidos.

Sob a hipótese de aprendizado (learning) por parte dos segurados, teríamos que no início da vigência eles são menos experientes e, portanto, apresentariam maior risco de sinistro. Então, observaríamos uma maior frequência de acidentes no início de cada contrato. Para minimizar este potencial viés, truncamos a amostra para segurados com idade superior a 25 anos, assumindo implicitamente que a partir desta idade o acréscimo marginal de aprendizado é desprezível. A quarta coluna da Tabela 3 apresenta a frequência de perdas ocorridas no mês de janeiro de 2004, porém, na subamostra de segurados com idade superior a 25 anos. Notamos que, mesmo após eliminar o possível viés causado pelo efeito aprendizado, a quantidade de perdas declaradas continua maior no início do contrato. ${ }^{8}$ Outro aspecto importante relacionado ao aprendizado do segurado diz respeito à aquisição de um veículo novo. Há duas possibilidades para classificar um automóvel como novo, pode ser no sentido de não ter sido adquirido por alguém anteriormente (zero $\mathrm{km}$ ) ou o segurado compra um modelo que ainda não conhece (mesmo este sendo um veículo seminovo). Infelizmente, nossa base de dados não contém o histórico de aquisições dos veículos, sendo assim, classificamos os carros de acordo com seu ano de fabricação. Com isso, truncamos novamente a amostra em janeiro de 2004, excluímos as apólices de segurados com menos de 25 anos e mantivemos apenas contratos com veículos cujo ano do modelo era inferior a 2003. Este resultado está na quinta coluna da Tabela 3. Note que mesmo fazendo essa truncagem, o fato estilizado ainda persiste. ${ }^{9}$

Destaca-se que dificilmente um segurado ao adquirir um novo modelo levaria um ano para aprender a dirigi-lo, na verdade em poucos meses ele consegue se acostumar com o novo veículo, assim o padrão esperado seria que após alguns meses a quantidade de acidentes declarados ficaria constante, mas isso não acontece como mostrado na quinta coluna da Tabela 3. Além disso, excluímos da base de dados as apólices que possuíam endosso, desta forma qualquer contrato que teve alguma alteração referente ao modelo do veículo não foi considerado.

\footnotetext{
8 Idem.

9 Idem.
} 
Porém, para discutir a hipótese de crescimento no número de apólices de seguro vendidas, é importante analisar a frequência dos novos contratos de no primeiro semestre de 2004, apresentada no Gráfico 1. De acordo com este gráfico, percebe-se que a quantidade de contratos cresce a cada mês, assim, ao fixarmos a amostra em determinado mês, temos que haverá mais apólices no primeiro mês de vigência do que no último. Desta maneira, ceteris paribus, haveria também uma maior frequência de sinistros declarados.

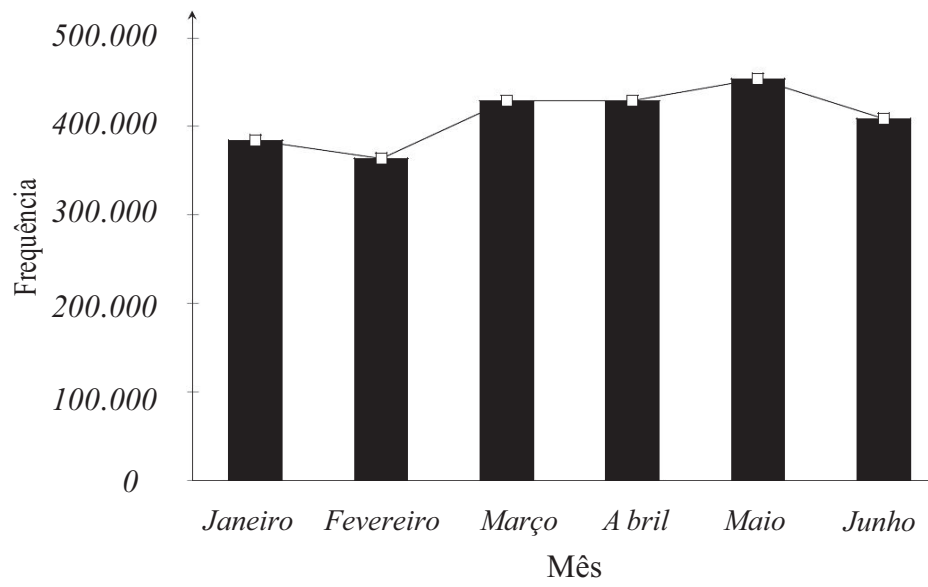

Gráfico 1 - Frequência dos novos contratos de seguros no primeiro semestre de 2004

Assim, para minimizar este potencial viés, truncamos novamente a amostra em janeiro de 2004 e consideramos apenas para os contratos cuja idade do segurado é maior que 25 anos e com classes de bônus positivas. Desta forma, dado que todo entrante no mercado, necessariamente, começa com classe de bônus igual a zero, garantimos que nossa amostra não contém contratos novos de seguro. Este resultado é apresentado na sexta coluna da Tabela 3, e percebe-se que o fato estilizado se mantém.

Os dados desta seção mostraram que a frequência de sinistros declarados é superior no início dos contratos de seguro. Não obstante, este fato estilizado mostrou-se bastante robusto a diferentes cortes amostrais que realizamos para isolar os efeitos da inflação, do aprendizado e do crescimento. Na próxima seção desenvolveremos um modelo teórico para racionalizar este fato estilizado. 


\section{Modelo recursivo de decisão do segurado}

Nesta seção apresentaremos uma versão modificada do modelo desenvolvido por Venezia e Levy (1980). É necessário ressaltar que fizemos duas alterações no modelo original ao considerar que (i) cada apólice de seguro fica vigente por dois períodos; e, (ii) podem ocorrer até 2 sinistros durante um mesmo contrato, sendo um em cada período. Originalmente os autores consideram que cada apólice dura um único período, e que ocorre apenas um acidente por contrato. A flexibilização destas hipóteses é importante, pois nos permitirá fazer uma análise que não está presente na literatura: comparar a pseudofranquia em diferentes períodos de um mesmo contrato.

Primeiramente, assuma que existam $J$ classes de risco e que cada uma está associada a um valor de prêmio distinto tal que $r_{1}<\cdots<r_{J-1}$. Assim, quanto maior a classe de risco, maior o preço do seguro. Além disso, considere que se o segurado está no rating de risco $r_{J}$ a seguradora não renovará seu contrato.

É importante destacar que se o segurado está na classe de risco $r_{j}$ e incorre em uma perda, ele terá a opção de declará-la ou não. Ao optar por acionar o seguro, ele será indenizado integralmente, mas no próximo período, o prêmio aumentará para $r_{j+1} \cdot{ }^{10}$ Por outro lado, caso opte por não comunicar à seguradora o acidente, ele deverá arcar sozinho com o valor da perda, porém, manter-se-á no mesmo rating de risco $r_{j}{ }^{11}$

Considere um consumidor que vive por $T$ períodos (finito). Podemos representar suas preferências pela seguinte função utilidade

$$
U\left(c_{1}, \ldots, c_{T}\right)=\sum_{t=1}^{T} \beta^{t} u\left(c_{t}\right)
$$

tal que $u($.$) é côncava e duas vezes diferenciável, c_{t}$ é o consumo no período $t, \beta \in(0,1)$ é o fator de desconto intertemporal.

\footnotetext{
${ }^{10}$ Por simplicidade, e sem perda de generalidade, supomos que o valor da franquia é zero. Ver Venezia e Levy (1979).

${ }^{11}$ A não redução da classe de risco não altera o resultado do modelo. Ver Venezia e Levy (1979).
} 
A cada período o consumidor recebe uma dotação exógena de renda $A_{t}$, que pode ser utilizada para pagar o prêmio do seguro e eventuais perdas, o restante é gasto exclusivamente com consumo no mesmo período. Considere que $F^{j}(t,$.$) é a função de distribuição das per-$ das, $Y$, de um segurado com classe de risco $r_{j}$ no momento $t$, e essa função tem suporte em $[0, M]$, tal que $M$ é suficientemente pequeno de modo que o segurado sempre terá renda para consumir.

Seja $z_{t}$ uma variável aleatória que representa as perdas não reembolsadas pela seguradora e $r(t)$ a classe de risco. Defina a função indicadora $I(t)$ de modo a assumir valor 1 caso $t$ seja um número ímpar e 0 se for número par. Assim, a utilidade descontada deste segurado é dada por

$$
U\left(c_{1}, \ldots, c_{T}\right)=\sum_{t=1}^{T} \beta^{t} u\left(A_{t}-z_{t}-I(t) r(t)\right)
$$

Note que a função indicadora $I(t)$ implica que, ao adquirir um contrato no período $t$ pelo preço $r(t)$, o segurado continuará assegurado no em $t+1$, sem que seja necessário pagar novamente o prêmio. Esta hipótese modifica o modelo original proposto por Venezia e Levy (1980), uma vez que permite que um contrato dure por dois períodos.

Definimos $V_{j t}(y)$ como a utilidade máxima esperada no período $t$ de um segurado com classe de risco $r_{j}$, e este adota uma estratégia de decisão ótima condicionada ao fato dele ter incorrido em uma perda no valor $y$. Além disso, $W_{j t}$ é o valor esperado de $V_{j t}(y)$, assim

$$
W_{j t}=E\left[V_{j t}(y)\right]
$$

É importante ressaltar que o operador esperança E é tomado em relação à distribuição $F^{j}(t,$.$) . Dado que o comportamento do$ segurado é baseado em uma estratégia ótima, o seu bem-estar é $W_{j t}$ (utilidade esperada descontada) no tempo $t$, considerando que o prêmio é $r_{j}$. Assim,

$$
W_{j t}=\sum_{\tau=t}^{T} \beta^{\tau-t} u\left(A_{\tau}-\tilde{z}_{\tau}-I(\tau) \tilde{r}(\tau) \mid r(\tau)=r_{j}\right)
$$


O consumidor inicia o período $t$ com uma renda exógena, $A_{t}$, e adquire um contrato de seguro ao preço $r_{j} \in\left\{r_{1}, \ldots, r_{J}\right\}$. Em seguida, sorteia uma perda $y$ da função de distribuição $F^{j}(t,$.$) e deve-$ rá decidir entre declará-la ou não. Caso opte por declarar, será indenizado integralmente e sua utilidade em $t$ será $u\left(A_{t}-I(t) r_{j}\right)$, mas no próximo período sua classe de risco será $r_{j+1}$ e a utilidade esperada será $W_{j+1, t+1}$. Logo, sua utilidade esperada em $t$ será $u\left(A_{t}-I(t) r_{j}\right)+\beta W_{j+1, t+1}$. Por outro lado, ao não declarar o sinistro, o segurado arcará sozinho com a perda, a utilidade presente e futura serão $u\left(A_{t}-I(t) r_{j}-y\right)$ e $W_{j, t+1}$, respectivamente, pois em $t+1$ o prêmio continuará $r_{j}$. Portanto, sua utilidade em $t$ será $u\left(A_{t}-I(t) r_{j}-y\right)+\beta W_{j, t+1}$.

Vale destacar que se o consumidor adquiriu uma apólice no período $t$ pelo preço $r_{j}$, ao não comunicar as perdas à seguradora durante a vigência do contrato (em $t$ e $t+1$ ), seu prêmio em $t+2$ continuará sendo $r_{j}$. Porém, ao declarar apenas um sinistro, o consumidor pagará $r_{j+1}$ em $t+2$ pela nova apólice. E, finalmente, se optar por acionar o seguro na ocorrência dos dois acidentes, seu prêmio em $t+2$ será $r_{j+2}$.

Dado que $V_{j t}(y)$ é a utilidade máxima esperada em cada período temos que

$$
\begin{gathered}
V_{j t}(y)=\max \left\{u\left(A_{t}-I(t) r_{j}-y\right)+\beta W_{j, t+1}, u\left(A_{t}-I(t) r_{j}\right)\right. \\
\left.+\beta W_{j+1, t+1}\right\}
\end{gathered}
$$

é a estratégia de decisão ótima do segurado pode ser obtida a partir da Equação (5). Além disso, uma perda, em determinado período, será declarada apenas se a seguinte condição for satisfeita

$$
u\left(A_{t}-I(t) r_{j}-y\right)+\beta W_{j, t+1}<u\left(A_{t}-I(t) r_{j}\right)+\beta W_{j+1, t+1}
$$

ou seja, se a utilidade esperada por não acionar o seguro for inferior a esta no caso em que o acidente é comunicado à seguradora. Dado que $u$ é côncava tem-se que $u^{\prime}>0$, então é possível estabelecer que existe um valor crítico, $y_{j t}^{*}$, tal que o seguro será acionado apenas se a perda exceder este valor. 
Com base na hipótese de continuidade de $u$, é possível determinar a pseudofranquia - o valor de perda superior a franquia que deixa o segurado indiferente entre declará-la ou não - que é igual a $y_{j t}^{*}$. Esta é definida de acordo com a seguinte equação

$$
u\left(A_{t}-I(t) r_{j}-y^{*}\right)=u\left(A_{t}-I(t) r_{j}\right)+\beta\left[W_{j+1, t+1}-W_{j, t+1}\right]
$$

Nota-se que, dado $t$, para obter $y_{j t}^{*}$ é necessário primeiramente calcular $W_{j t}$ conforme segue

$$
\begin{aligned}
W_{j t} & =E\left[V_{j t}(y)\right], \\
& =E\left(\max \left\{u\left(A_{t}-I(t) r_{j}-Y\right)+\beta W_{j, t+1}, u\left(A_{t}-I(t) r_{j}\right)+\beta W_{j+1, t+1}\right\}\right), \\
& =E\left(\beta W_{j, t+1}+\max \left\{u\left(A_{t}-I(t) r_{j}-Y\right), s\right\}\right), \\
& =\beta W_{j, t+1}+E\left(\max \left\{u\left(A_{t}-I(t) r_{j}-Y\right), s\right\}\right), \\
& =\beta W_{j, t+1}+\int_{0}^{y^{*}}\left[u\left(A_{t}-I(t) r_{j}-y\right)\right] d F^{j}(t, y)+\int_{y^{*}}^{M} s d F^{j}(t, y),
\end{aligned}
$$

tal que $\mathrm{q}^{12}$

$$
\begin{aligned}
& s=u\left(A_{t}-I(t) r_{j}\right)+\beta\left[W_{j+1, t+1}-W_{j, t+1}\right], \\
& y^{*} \text { satisfaz: } u\left(A_{t}-I(t) r_{j}-y^{*}\right)=s
\end{aligned}
$$

Ao somar e subtrair a seguinte expressão em (8)

$$
\int_{0}^{y^{*}} s d F(t, y)
$$

obtemos

$$
\begin{aligned}
& W_{j t}=u\left(A_{t}-I(t) r_{j}\right)+\beta W_{j+1, t+1}+\int_{0}^{y^{*}}\left[u\left(A_{t}-I(t) r_{j}-y\right)-s\right] d F^{j}(t, y), \\
& j=1, \ldots, J-1 e t=1, \ldots, T-1
\end{aligned}
$$

\footnotetext{
${ }^{12}$ Apenas para facilitar a notação os índices $j$ e $t$ de $s, y^{*}$, e $F($.$) foram omitidos.$
} 
tal que $s$ e $y^{*}$ foram definidos em (9) e as pseudofranquias são determinadas por $y_{j t}^{*} s$.

Embora aparentemente complexa, a Equação (11) traz um resultado bastante simples. A primeira parte desta equação apresenta a utilidade esperada por declarar uma perda (quando $y>y^{*}$ ) dada por $u\left(A_{t}-I(t) r_{j}\right)+\beta W_{j+1, t+1}$. Já a segunda parte apresenta o benefício marginal esperado por não declarar um sinistro, mesmo que este seja superior ao valor da franquia (quando $y \in\left[0, y^{*}\right]$ ). Porém, vale ressaltar que se $y=0$ não há ganho adicional em não comunicar o acidente à seguradora, portanto, a riqueza esperada é definida apenas pela utilidade ao declarar. Por outro lado, quanto maior o valor de $y^{*}$, maior é a riqueza esperada, pois maior será o ganho ao não declarar o sinistro. Além disso, note que $W_{j t}$ tem como limite inferior a utilidade esperada ao declarar.

Dessa forma, se no momento $t$ o segurado sorteia uma perda, ele acionará o seguro apenas se essa for superior a $y_{j t}^{*}$, dado que possui uma classe de risco $r_{j}$. Finalmente, $W_{j T} s$ pode ser facilmente computada, uma vez que no período $T$ o segurado sempre declara o acidente, pois ele não pode ser penalizado por isso. Assim,

$$
W_{j T}=u\left(A_{T}\right), W_{J T}=E\left(u\left(A_{T}-Y\right)\right), j=1, \ldots, J-1
$$

Com isso calculamos todos os demais valores de $W_{j T} s$ e utilizando (12), (11) e (9) computamos $y_{j t}^{*} s$ para $t<T$ recursivamente começando por $t=T-1$.

Solução Analítica Conforme vimos, a Equação (9) define implicitamente a pseudofranquia a partir da condição de indiferença entre declarar ou não uma perda. Logo, para obter uma solução explícita seria necessário supor alguma função utilidade específica, o que nos faria perder em generalidade. Para contornar este problema, reescreveremos a Equação (9) a partir do coeficiente de aversão absoluto ao risco do segurado. Para tal, considere a seguinte expansão de Taylor da função $u($.$) no ponto A_{t}-I(t) r_{j}-y$

$$
u\left(A_{t}-I(t) r_{j}-y^{*}\right) \approx u\left(A_{t}-I(t) r_{j}\right)-y^{*} u^{\prime}\left(A_{t}-I(t) r_{j}\right)+\frac{y^{* 2}}{2} u^{\prime \prime}\left(A_{t}-I(t) r_{j}\right)
$$


Substituindo (13) em (9) temos o seguinte resultado

$$
-\frac{u^{\prime \prime}\left(A_{t}-I(t) r_{j}\right)}{u^{\prime}\left(A_{t}-I(t) r_{j}\right)}=-\frac{2}{y^{*}}+\frac{2 \beta\left[W_{j, t+1}-W_{j+1, t+1}\right]}{y^{* 2} u^{\prime}\left(A_{t}-I(t) r_{j}\right)}+\epsilon
$$

A Equação (14) nos oferece uma solução analítica para o problema de escolha do segurado. Do lado esquerdo da igualdade temos o coeficiente de aversão absoluta ao risco, que por sua vez não depende da pseudofranquia, mas apenas de váriaveis exógenas. Do lado direito da igualdade temos a pseudofranquia $y^{*}$ e um segundo termo que depende da diferença entre as riquezas esperadas no período $t+1$, dada por $W_{j, t+1}-W_{j+1, t+1}$.

Dada a hipótese de que um contrato dura por dois períodos, o segurado pagará o prêmio em períodos intercalados. Suponha que o indivíduo adquire um seguro no período $t$ e declare uma perda neste período. Em $t+1$ o mesmo contrato continuará vigente sem que haja cobrança de novo prêmio. $\mathrm{Na}$ verdade, o aumento no valor da apólice só virá no em quando um novo contrato for assinado. Sendo assim, este acréscimo de prêmio será incorporado pelo termo $W_{j+1, t+1}$ com "carência" de um período. Neste caso, teremos $W_{j, t+1}-W_{j+1, t+1}>0$.

Agora, suponha que o segurado pague o prêmio no período $t$, não declare perda neste período, mas declare em $t+1$. Neste caso, o segurado sofrerá o acréscimo no valor do contrato logo no período seguinte. Assim, sua riqueza esperada dada por $W_{j+1, t+2}$ será ainda menor do que no caso do parágrafo anterior. Portanto, teremos $W_{j, t+2}-W_{j+1, t+2}>W_{j, t+1}-W_{j+1, t+1}>0$.

Finalmente, agora podemos utilizar a Equação (14) para analisar a trajetória da pseudofranquia ao longo do tempo. Dada uma aversão absoluta ao risco, a Equação (14) nos diz que no segundo período de um contrato a pseudofranquia, $y^{*}$, será maior, pois $W_{j, t+2}-W_{j+1, t+2}>W_{j, t+1}-W_{j+1, t+1}>0$. Em outras palavras, demonstramos que dentro de um mesmo contrato a pseudofranquia aumenta do primeiro para o segundo período. Este é o principal resultado do nosso modelo, pois explica justamente nosso fato estilizado. 
Mas, o que acontece com a pseudofranquia entre diferentes contratos? Dado que nosso modelo tem finitos períodos, à medida que o tempo passa o termo $W_{j, t+1}-W_{j+1, t+1}$ converge para zero. Logo, entre diferentes contratos a pseudofranquia diminui. Este resultado é o mesmo encontrado no modelo original de Venezia e Levy (1980). Em resumo, nosso modelo modificado apresenta uma trajetória oscilatória para pseudofranquia, aumentando intracontratos e diminuindo intercontratos.

\section{Exemplo numérico}

A partir do nosso modelo, reconstruímos o mesmo exemplo numérico desenvolvido por Venezia e Levy (1980). Com ele será possível observar o comportamento da pseudofranquia intra e intercontratos. Além disso, este exercício foi realizado tanto para segurados neutros quanto para avessos ao risco. Vale enfatizar que escolhemos apresentar este exemplo numérico apenas para fazer o contraponto ao artigo original, portanto, os números encontrados não têm qualquer aplicação empírica.

Suponha que a dotação exógena de renda é $A=20$ para todo $t \leq T, \beta \epsilon(0,1)$ e que o horizonte de tempo é finito tal que a apólice pode ser renovada 5 vezes, ou seja, $T=12$. Além disso, há 5 classes de risco definidas por

$$
r_{j}=(1,2)^{j} E(Y)
$$

Adicionalmente, admita que a função de distribuição de $Y$, variável aleatória que representa as perdas do segurado, não se altera com mudanças em $t$ ou $j$ e essa foi definida de acordo com a variância de 7,844 como mostrado na Tabela 4 . 
Tabela 4 - Função de distribuição das perdas

\begin{tabular}{cc}
\hline$y$ & $p(y)$ \\
\hline 0 & 0,4063 \\
1 & 0,0625 \\
2 & 0,0156 \\
3 & 0,0313 \\
4 & 0,0156 \\
5 & 0,0625 \\
6 & 0,4063 \\
\hline
\end{tabular}

Fonte: Venezia e Levy (1980).

A Figura 1 contém os resultados deste exemplo para segurados neutros ao risco. Esta foi baseada na Tabela 6 do Anexo. A partir desta é possível analisar a riqueza do segurado e a pseudofranquia para cada $t$ e $r_{j}$. Já a Figura 2 apresenta estes resultados para um segurado avesso ao risco, os dados estão na Tabela 7 do Anexo.
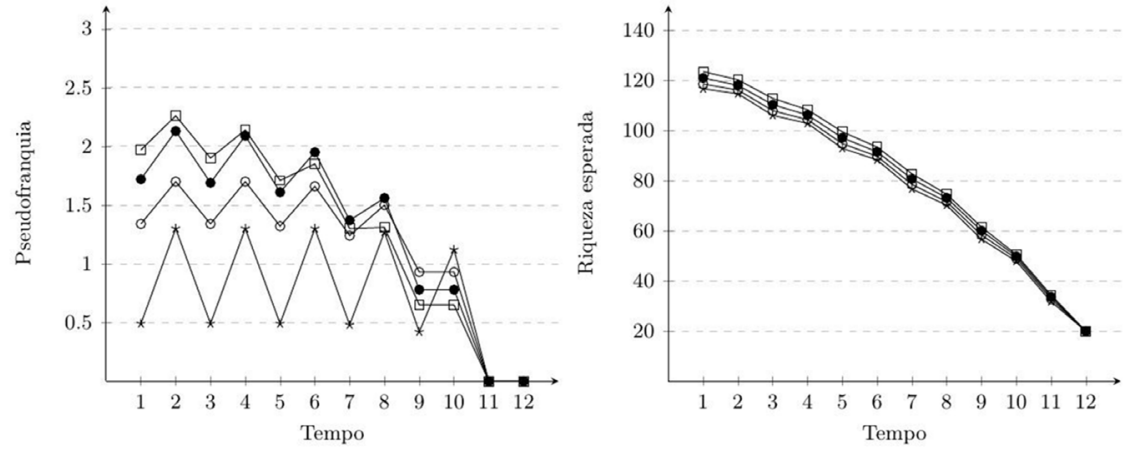

Figura 1 - Evolução da pseudofranquia e da riqueza esperada ao longo do tempo para cada classe de risco $j$, considerando um indivíduo neutro ao risco 

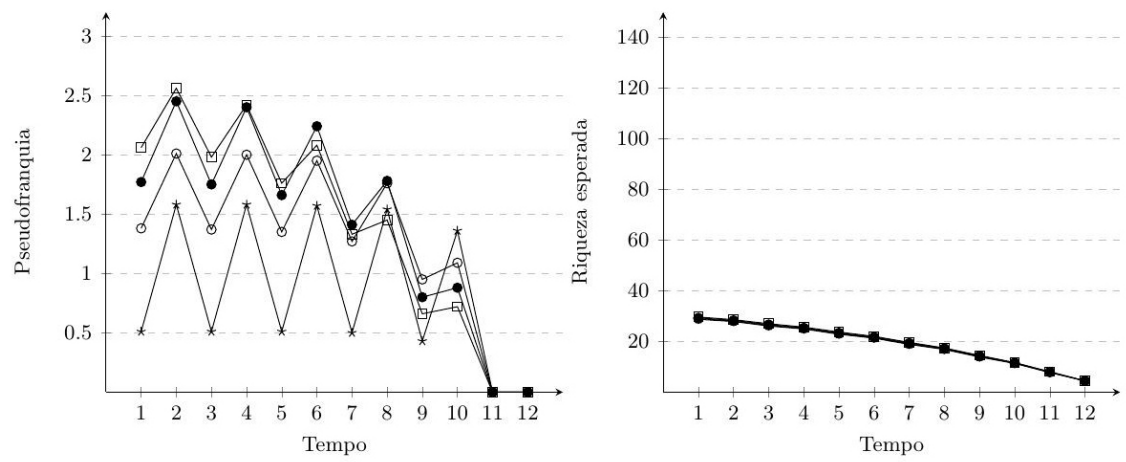

Figura 2 - Evolução da pseudofranquia e da riqueza esperada ao longo do tempo para cada classe de risco $j$, considerando um indivíduo avesso ao risco

A trajetória decrescente da pseudofranquia já era prevista no modelo original proposto por Venezia e Levy (1980). A explicação desse resultado é simples: ao declarar um sinistro, o segurado arca com um aumento de prêmio que se perpetua até o último período. Logo, quanto mais próximo estiver do último período, menor será a desutilidade de acionar o seguro e, portanto, menor a pseudofranquia.

A novidade em nosso modelo é a trajetória oscilatória. Embora a pseudofranquia decresca no longo prazo, ela cresce do primeiro para o segundo período de um mesmo contrato. A explicação deste resultado também é simples: supondo que ocorreu uma perda no primeiro período e dado que não se paga prêmio no segundo, o segurado ganha um período de carência antes de arcar com o aumento de prêmio. Esta carência o incentiva a declarar perdas menores no primeiro período de um determinado contrato.

De acordo com a Figura 2 percebe-se que à medida que aumenta a aversão ao risco do segurado, menor é $y^{*}$ no primeiro período do contrato e maior a pseudofranquia no segundo período, quando comparamos com um indivíduo neutro ao risco. Isso ocorre porque o objetivo do segurado é evitar aumentos no valor do prêmio, por mais que ele esteja mais propenso a acionar o seguro no primeiro período (menor $y^{*}$ ), ele sabe que o valor do prêmio não diminui e ainda arcará com o aumento do preço do seguro em caso de declaração de sinistro até a última apólice. 
Além disso, o indivíduo quer ter um nível de consumo constante ao longo do tempo. Assim, a pseudofranquia do segundo período do contrato é utilizada como mecanismo de proteção em relação ao próximo período, no qual há o pagamento do prêmio e pode ser que o segurado pague até $r_{j+2}$ dependendo das decisões tomadas, por isso apresenta valor mais elevado. Este resultado racionaliza o fato estilizado apresentado na Seção 3 e nos fornece uma previsão testável para o modelo.

\section{Testando o modelo}

Existe uma relação direta entre o fato estilizado observado nos dados e a previsão do modelo teórico. Ao demonstrar que a pseudofranquia é menor no início do contrato, o modelo prevê que pode haver um número maior de acidentes sendo declaradas neste período. Além disso, estas perdas terão um valor médio menor justamente em função da menor pseudofranquia. Sendo assim, o modelo nos fornece uma previsão testável direta: o valor esperado das perdas declaradas deve ser menor no começo da apólice.

Para testar esta previsão, regredimos o logaritmo do valor das indenizações contra algumas medidas de tempo de vigência contratual, além de outras variáveis explicativas, tais como: idade do segurado (corresponde a quantidade de anos do segurado até a data de início da apólice), tipo de franquia (variável binária que assume valor 1 se a franquia é normal ou 0 caso esta seja reduzida), prêmio (valor da apólice de seguro do carro), valor da franquia (variável que corresponde ao valor, em reais, da franquia contratada), regiões (variáveis binárias que indicam os locais onde veículo circula), gênero (variável binária que assume valor 1 se este for do sexo masculino), porcentagem de corretagem (representa a taxa de corretagem sobre o prêmio do seguro recebida pelo corretor como remuneração pela venda do contrato), porcentagem de bônus (corresponde ao percentual de desconto sobre o prêmio total do casco), importância segurada (equivale ao valor do automóvel que foi segurado), ano e modelo do carro. Além disso, consideramos a subamostra na qual foram excluídos os entrantes no mercado e os segurados com idade inferior a 25 anos. 
É importante destacar que o valor da indenização pode ser maior no final da vigência do contrato em função da inflação. Para lidar com esse viés, a alternativa seria deflacionar essa variável para um único período, porém não se sabe ao certo qual deve ser esse índice e como a inflação afeta a precificação das seguradoras. Assim, para lidar com esse problema, utilizamos o logaritmo da indenização como variável dependente e incluímos dummies para os meses do primeiro semestre de 2004 para capturar as variações relativas à passagem do tempo.

Para verificar se o timing é um fator relevante na decisão do segurado, elaboramos 3 regressões lineares múltiplas estimadas por Mínimos Quadrados Ordinários. A primeira divide o tempo de vigência do contrato em bimestres e, de acordo com os resultados da segunda coluna da Tabela 5, infere-se que os coeficientes do terceiro, do quarto e do sexto bimestres são positivos e estatisticamente significantes a 1,5 e $10 \%$, respectivamente. Nesta especificação obtivemos que, em média, o valor das perdas declaradas é aproximadamente $3,22 \%$ maior no terceiro bimestre, $2,38 \%$ maior no quarto bimestre e 2,11\% maior no sexto bimestre quando comparadas ao primeiro bimestre. Na segunda especificação foram usadas variáveis dummies para cada trimestre de vigência do contrato e, de acordo com a quarta coluna da Tabela 5, nota-se que os coeficientes do segundo e do terceiro trimestre também são positivos e ambos são significantes a $5 \%$. Porém, a terceira especificação divide o tempo de vigência do contrato em quadrimestres. Podemos observar que o coeficiente da dummy do segundo quadrimestre é positivo e estatisticamente significante a $1 \%$. Infere-se que o valor das perdas são aproximadamente 2,45\% maiores no segundo quadrimestre, em média. Sendo assim, todos os resultados apresentados na Tabela 5 corroboram a previsão do modelo teórico. 
Tabela 5 - Regressões do logaritmo das indenizações

\begin{tabular}{|c|c|c|c|c|c|}
\hline \multicolumn{2}{|c|}{ Regressão 1} & \multicolumn{2}{|c|}{ Regressão 2} & \multicolumn{2}{|c|}{ Regressão 3} \\
\hline $2^{\circ}$ Bimestre & $\begin{array}{c}0,0076 \\
(0,0105)\end{array}$ & $2^{\circ}$ Trimestre & $\begin{array}{l}0,0221^{* *} \\
(0,0087)\end{array}$ & $2^{\circ}$ Quadrimestre & $\begin{array}{r}0,0245^{\star * \star} \\
(0,0076)\end{array}$ \\
\hline $3^{\circ}$ Bimestre & $\begin{array}{c}0,0322^{\star * *} \\
(0,0106)\end{array}$ & $3^{\circ}$ Trimestre & $\begin{array}{l}0,0198^{* *} \\
(0,0089)\end{array}$ & $3^{\circ}$ Quadrimestre & $\begin{array}{l}0,0119 \\
(0,008)\end{array}$ \\
\hline $4^{\circ}$ Bimestre & $\begin{array}{l}0,0238^{\star *} \\
(0,0107)\end{array}$ & $4^{\circ}$ Trimestre & $\begin{array}{l}0,0122 \\
(0,009)\end{array}$ & & \\
\hline $5^{\circ}$ Bimestre & $\begin{array}{l}0,0101 \\
(0,011)\end{array}$ & & & & \\
\hline $6^{\circ}$ Bimestre & $\begin{array}{l}0,0211^{*} \\
(0,011)\end{array}$ & & & & \\
\hline Amostra & 97.234 & & 97.234 & & 97.234 \\
\hline $\mathrm{R}^{2}$ & $6,29 \%$ & & $6,28 \%$ & & $6,29 \%$ \\
\hline
\end{tabular}

Significante a 1\%; Desvios-padrão entre parênteses; Regressões incluem o termo constante. As demais variáveis explicativas foram omitidas por simplicidade de exposição.

Fonte: Elaboração própria.

\section{Conclusão}

Neste artigo apresentamos uma versão modificada do modelo recursivo de Venezia e Levy (1980). A hipótese adotada permitiu que um mesmo contrato durasse por dois períodos, o que resultou em uma nova previsão teórica capaz de explicar porque se observa uma frequência maior de perdas comunicadas à seguradora nos primeiros períodos dos contratos. Ao mesmo tempo, o modelo manteve a previsão do modelo original: ao longo do tempo, a quantidade de sinistros declarados deve aumentar à medida que se aproxima da última apólice que será adquirida pelo segurado.

Intuitivamente, o resultado de nosso modelo é bastante simples. Ao supor que uma perda ocorreu no primeiro período, e dado que não se paga prêmio no segundo, o segurado tem um período de carência antes de arcar com o aumento de prêmio. Isto o incentiva a declarar perdas menores no primeiro período de um determinado contrato. Sendo assim, o modelo tem a vantagem de gerar uma previsão testável direta. Se a pseudofranquia é menor no início do contrato, então 
acidentes de menor valor devem ser declaradas nos primeiros períodos da apólice, o que aumenta a frequência de perdas observadas.

Levando o modelo aos dados, testamos se o timing de ocorrência dos sinistros é relevante na estratégia ótima de decisão do segurado diante do trade-off entre declarar ou não suas perdas. Verificamos que os valores dos acidentes declarados são, em média, menores nos primeiros meses do contrato. Obtivemos que no segundo trimestre de vigência contratual o valor médio das perdas comunicadas à seguradora é aproximadamente $2,21 \%$ maior em relação ao primeiro trimestre. Além disso, no segundo quadrimestre as perdas declaradas são aproximadamente $2,45 \%$ maiores do que no primeiro. Adicionalmente, estas informações podem ser utilizadas pelas seguradoras para calibrar seus sistemas de precificação, com impacto direto em seus índices de sinistralidade via pseudofranquia.

\section{Referências}

BRAUN, M., et al. "Modeling the "Pseudodeductible" in Insurance Claims Decisions." Management Science. Vol. 52, No. 8, pp 1258-1272, 2006.

DE LEVE, G. e WEEDA, P.J. “Driving with Markov-programming”. The ASTIN Bulletin. No. 5, pp 62-86, 1968.

DELLAERT, N. P., et al. “Optimal Claim Behaviour for Third-Party Liability Insurances or To claim or not to claim: that is the question." Insurance: Mathematics and Economics. Vol. 9, pp 59-76, 1990.

DE PRIL, N. “Optimal Claim Decisions for a Bonus-Malus System: a Continuous Approach”. International Journal for Actuarial Studies in Non-life Insurance and Risk Theory. Vol. 10, pp 215-222, 1979.

DE PRIL, N. e GOOVAERTS, M. "Bounds for the Optimal Critical Claim Size of a Bonus System". Insurance: Mathematics and Economics. Vol. 2. No. 1, pp 27-32, 1983.

GRENANDER, U. "Some Remarks on Bonus Systems in Automobile Insurance”. Skandinavisk Aktuarietidskrift, Vol 40, pp 180-198, 1957.

HASTINGS, N.A.J. “Optimal Claiming on Vehicle Insurance”. Operational Research Quarterly. Vol. 27. No. 4, pp 805-813, 1976.

KERR, D. A. "Exploring the role of pseudodeductible in auto insurance claims reporting". Journal of Insurance Issues. Vol 35, No.1, pp 44-72, 2012.

KOLDERMAN, J. e VOLGENANT, A. "Optimal Claiming in an Auto-mobile Insurance System with Bonus-Malus Structure". Journal of the Operatio-nal Research Society. Vol. 36. No. 3, pp 239247, 1985.

LANZENAUER, C. H. "Entscheidungsregeln fur das optimale Verhalten in der Kraftfahrtversicherung". Unternemensforschung. 13, pp. 193-204, 1969.

LANZENAUER, C. H. "Decision problems in the Canadian automobile insurance systems". Journal of Risk and Insurance. Vol 39, No. 1, pp 79-92, 1972a. 
LANZENAUER, C. H. "The effects of the insurees' decisions on the insurer's profit". Proceedings in Operations Research. Physica Verlag, Heidelberg, pp 399-421, 1972b.

LANZENAUER, C. H. "Optimal Claim Decisions by Policyholders in Automobile Insurance with Merit-Rating Structures”. Operations Research. Vol. 22, No. 5, pp. 979-990, 1974.

LEMAIRE, J. "Driver versus Company”. Scandinavian Actuarial Journal. pp 209-219, 1996.

NORBERG, R. "A Credibility Theory for Automobile Bonus System”. Scandinavian Actuarial Journal, 92-109, 1976

NORMAN, J.M. e SHEARN, D.C.S. "Optimal Claiming on Vehicle Insurance Revisited". Journal of the Operational Research Society. Vol. 31. No. 2, pp 181-186, 1980.

VENEZIA, I. “Aspects of Optimal Automobile Insurance". The Journal of Risk and Insurance. Vol 51 No 1, pp 63-79, 1984.

VENEZIA, I. e LEVY, H. "Optimal Claim in Automobile Insurance". Review of Economic Studies. pp. 539-549, 1980.

VENEZIA, I. e LEVY, H. "Optimal Claim in Automobile Insurance". (Discussion Paper 79-2, Department of Economics, University of Rochester), 1979.

\section{Anexo}

Tabela 6 - As riquezas e pseudofranquias do segurado neutro ao risco como função de $t$ e $j$

\begin{tabular}{ccccccccc}
\hline \multicolumn{3}{c}{$j=1$} & \multicolumn{3}{c}{$j=2$} & \multicolumn{2}{c}{$j=3$} & \multicolumn{2}{c}{$j=4$} \\
$\mathrm{t}$ & $W_{1, t}$ & $y_{1, t}$ & $W_{2, t}$ & $y_{2, t}$ & $W_{3, t}$ & $y_{3, t}$ & $W_{4, t}$ & $y_{4, t}$ \\
\hline 1 & 123,59 & 1,97 & 121,04 & 1,72 & 118,66 & 1,34 & 116,76 & 0,49 \\
2 & 120,34 & 2,26 & 118,15 & 2,13 & 116,24 & 1,70 & 114,75 & 1,30 \\
3 & 112,89 & 1,90 & 110,38 & 1,69 & 108,01 & 1,34 & 106,12 & 0,49 \\
4 & 108,40 & 2,14 & 106,29 & 2,09 & 104,41 & 1,70 & 102,92 & 1,30 \\
5 & 99,56 & 1,71 & 97,18 & 1,61 & 94,85 & 1,32 & 92,97 & 0,49 \\
6 & 93,48 & 1,85 & 91,57 & 1,95 & 89,78 & 1,66 & 88,32 & 1,30 \\
7 & 82,81 & 1,30 & 80,75 & 1,37 & 78,58 & 1,24 & 76,74 & 0,48 \\
8 & 74,62 & 1,31 & 73,18 & 1,56 & 71,65 & 1,50 & 70,27 & 1,27 \\
9 & 61,53 & 0,65 & 60,08 & 0,78 & 58,34 & 0,93 & 56,68 & 0,42 \\
10 & 50,57 & 0,65 & 49,85 & 0,78 & 48,98 & 0,93 & 47,94 & 1,12 \\
11 & 34,40 & 0,00 & 33,68 & 0,00 & 32,82 & 0,00 & 31,78 & 0,00 \\
12 & 20,00 & - & 20,00 & - & 20,00 & - & 20,00 & - \\
\hline
\end{tabular}

Fonte: Elaboração própia. 
Tabela 7 - As riquezas e pseudofranquias do segurado avesso ao risco como função de $t$ e $j$

\begin{tabular}{|c|c|c|c|c|c|c|c|c|}
\hline \multirow[b]{2}{*}{$\mathrm{t}$} & \multicolumn{2}{|c|}{$j=1$} & \multicolumn{2}{|c|}{$j=2$} & \multicolumn{2}{|c|}{$j=3$} & \multicolumn{2}{|c|}{$j=4$} \\
\hline & $W_{1, t}$ & $y_{1, t}$ & $W_{2, t}$ & $y_{2, t}$ & $W_{3, t}$ & $y_{3, t}$ & $W_{4, t}$ & $y_{4, t}$ \\
\hline 1 & 29,68 & 2,06 & 29,34 & 1,77 & 29,03 & 1,38 & 28,77 & 0,51 \\
\hline 2 & 28,64 & 2,56 & 28,35 & 2,45 & 28,09 & 2,01 & 27,89 & 1,58 \\
\hline 3 & 27,03 & 1,98 & 26,70 & 1,75 & 26,39 & 1,37 & 26,13 & 0,51 \\
\hline 4 & 25,69 & 2,42 & 25,41 & 2,40 & 25,16 & 2,00 & 24,96 & 1,58 \\
\hline 5 & 23,75 & 1,76 & 23,44 & 1,66 & 23,13 & 1,35 & 22,88 & 0,51 \\
\hline 6 & 22,03 & 2,08 & 21,78 & 2,24 & 21,54 & 1,95 & 21,34 & 1,57 \\
\hline 7 & 19,66 & 1,33 & 19,39 & 1,41 & 19,10 & 1,27 & 18,86 & 0,50 \\
\hline 8 & 17,45 & 1,45 & 17,26 & 1,78 & 17,06 & 1,76 & 16,87 & 1,54 \\
\hline 9 & 14,52 & 0,66 & 14,34 & 0,80 & 14,11 & 0,95 & 13,89 & 0,43 \\
\hline 10 & 11,69 & 0,72 & 11,60 & 0,88 & 11,49 & 1,09 & 11,35 & 1,36 \\
\hline 11 & 8,07 & 0,00 & 7,98 & 0,00 & 7,87 & 0,00 & 7,74 & 0,00 \\
\hline 12 & 4,47 & - & 4,47 & 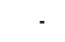 & 4,47 & - & 4,47 & - \\
\hline
\end{tabular}

Fonte: Elaboração própia. 